\title{
Study on Ultrasound Diagnosis of Common Abdominal Obstetrics and Gynecology
}

\author{
Xiaojun Liu ${ }^{1}$, Lili Xuan*,1 \\ ${ }^{1}$ China-Japan Union hospital of Jilin University, Changchun, Jilin, 130033 \\ *corresponding author
}

Keywords: Ultrasound diagnosis; Abdominal Obstestrics; Gynecology

\begin{abstract}
Obstetrics and Gynecology acute abdomen is a common disease in the clinic. Patients generally have an urgent performance at the time of treatment, the condition develops rapidly, and early clinical manifestations are occult, but the onset is rapid and life-threatening. Therefore, clinicians are required to make prompt and accurate diagnosis and intervention. Among various inspection measures, ultrasound examination has become the preferred method for the examination of acute abdomen in obstetrics and gynecology because of its non-invasive, rapid, and effective advantages. Most of the Obstetrics and Gynecology acute abdomen diseases are related to bleeding. If they cannot be diagnosed and treated in time, they will seriously endanger the lives of patients. Therefore, how to make accurate diagnosis and treatment of obstetrics and gynecology has an important clinical significance [1]. With the continuous development of information technology and continuous improvement of medical examination methods, ultrasound diagnosis has become more and more widely used in the field of obstetrics and gynecology, and has played a significant role in the clinical practice of obstetrics and gynecology type acute abdomen, significantly reducing The mortality rate of obstetrics and gynecology patients with acute abdomen [2]. This paper first briefly introduces the clinical features and sonographic features of various common obstetric and gynecological acute abdomen diseases, and then analyzes the diagnostic value of obstetrics and gynecology and acute abdomen. Its purpose is to explain the current status and future of ultrasound diagnosis in obstetrics and gynecology.
\end{abstract}

\section{Introduction}

Obstetrics and Gynecology acute abdomen is a common clinical disease. In recent years, the number of patients suffering from gynecologic acute abdomen has been increasing, and the incidence rate has been increasing year by year. Gynecological acute abdomen often has a more acute onset, a more complicated etiology, and a rapidly progressing condition. The clinical diagnosis is relatively difficult and poses a serious threat to the patient's health $[1,2]$. In the early stage of patient's onset, early and accurate diagnosis, and take active and effective treatment measures, patients often have a good prognosis. Therefore, the diagnostic study of obstetrics and gynecology acute abdomen is of great value. This study selected 126 cases of acute abdomen patients admitted to our hospital. All patients underwent ultrasound examinations before surgery to observe the diagnostic results of ultrasound diagnosis. The results are reported below.

\section{Clinical Features and Audiovisual Features of Various Abdomen and Gynecology Acute Abdomen}

Abortion is one of the more common causes of acute abdomen in obstetrics and gynecology. Abortion is roughly divided into threatened abortion, inevitable miscarriage, and incomplete abortion. The main symptoms are abdominal pain, bleeding, etc. [4 - 6]. Threatened abortion visible intrauterine gestational sac, capsule seen in the capsule and the heart beat, indicating embryo survival; if the embryonic fetal heart beat is too low, that is, the tendency of miscarriage; fetal heart beat was scintillation blood flow signal, high echo chorion There is still a low resistance to 
trophoblast blood flow. Inevitably, miscarriage may show signs of deformed shrinkage of the gestational sac, detachment of the edge defect, and downward movement of the position; if the gestational sac does not peel off, blood flow of the trophoblast with low resistance may still be recorded. Incomplete abortion shows that the uterus is enlarged or full, there are loose light bands or loose light masses in the uterine cavity; Irregular, uneven mass echoes in the uterine cavity, or hypoechoic or hyperechoic, should be differentiated from hydatidiform mole [7].

Ectopic pregnancy is commonly known as ectopic pregnancy. With the change of modern living environment and lifestyle, the incidence of ectopic pregnancy has been increasing year by year, and because of its unpredictable symptoms, it is very easy to endanger the lives of patients. In general, patients will experience sudden and severe abdominal pain and even shock coma. Without timely and effective medical intervention, they may lose their lives due to excessive blood loss. Ectopics generally include ovarian pregnancies, tubal pregnancies, intra-abdominal pregnancies, and cervical pregnancies. About 95\% of them occur in the fallopian tubes [8]. At the time of ultrasound diagnosis, we can only observe that the uterus is small in size, and no cystic echography can be found in the uterine cavity. However, in the pelvic cavity, there are mixed echogenic solid masses, which can be unevenly high and low echoes. The shape of the mass is generally irregular, and is closely related to the uterus. Occasionally, there may be signs of separation of the endometrium to form a pseudo-gestational sac, which should be distinguished from intrauterine pregnancy. The ectopic pregnancy has a long rupture time and a large amount of bleeding. In patients with ultrasound, there will be intestinal and uterus floating. However, in clinical practice, patients with abnormal ultrasound images and inaccurate clinical information (such as irregular menstruation and no history of menopause) are sometimes encountered. The diagnosis of ectopic pregnancy cannot be released. At this time, the clinician is required to be in an abnormal position. Pregnancy, rupture of the corpus luteum, pelvic inflammatory mass, endometriosis, and ovarian cyst torsion [9] are carefully distinguished and diagnosed, although blood B-HCG can be used as a diagnostic indicator with high reference Value, but wait for longer results will delay the diagnosis and treatment of acute abdomen [1 0]. Using C D F I technology, we can also observe nourishing blood flow signals in and around pelvic masses during ectopic pregnancy examinations, so ultrasound plays an increasingly important role in the diagnosis and treatment of ectopic pregnancy.

\section{Ultrasonic Diagnosis in Obstetrics and Gynecology}

For obstetrics and gynecology acute abdomen, the advantage of ultrasound examination is that it is simple, safe, intuitive, non-invasive, inexpensive, easy to follow, and the differential diagnosis is rapid and accurate. It can be used as the first choice for clinical examination [18]. With the advancement of medical science and the improvement of inspection techniques, ultrasonography can accurately diagnose the type of acute abdomen in obstetrics and gynecology and accurately assess the degree of injury, such as threatened abortion, inevitable miscarriage, incomplete abortion, ectopic pregnancy, type, The amount of bleeding, the type and timing of the umbilical cord around the neck and around the body, the location of uterine perforation, the location and size of the uterine rupture, the degree of rupture of the uterus during pregnancy, and the degree of placenta previa were greatly improved in clinical status. Not only that, ultrasound can also accurately screen between non-obstetric and gynecological acute abdomen and gynecologic acute abdomen, abdominal pain caused by other systems (such as urethra or lower ureteral stones, appendicitis, intestinal lesions, etc. [1 9]) It is also possible to make a definitive diagnosis and assist the clinician in the rapid and accurate diagnosis and treatment of the patient.

\section{Ultrasound Results and Methods}

The cause of acute abdominal disease in obstetrics and gynecology is more common in abortions. Menopause, abdominal cramps, and vaginal bleeding are the main symptoms of abortion. However, there are no specificities in the stages of abortion development. Clinically determined treatment plans have certain blindness. Ultrasound can be judged by observing the size and shape of the 
gestational sac with or without embryos and fetal heart beats. Sonographic features: (1) Threatened abortion: The size of the uterus and the gestational sac corresponds to the menopause month, and the position of the gestational sac is normal or low. The gestational sac was shown in the uterus. In the capsule, germs and fetal heartbeats were seen to be pulsed. This indicates that the germ has survived and can continue to gestation. If the gestational sac is ruled, no germs and heartbeats are pulsated and there is less vaginal bleeding. Observed after 1 week B-ultrasound, If you still have not seen germ, and the gestational sac deformed, you can consider termination of pregnancy. (2) Abortion is unavoidable: Ultrasound shows that the size of the gestational sac does not match the gestational age, the deformation is irregular, the location of the gestational sac moves down, and the heart and the heart in the capsule disappear. Withered gestational sac is characterized by a circular or incomplete circular echo-free zone in the uterine cavity. The wall echo is clear or unclear. There are no fetal buds in the gestational sac and there is no fetal heartbeat. Observe that the gestational sac can increase and the placenta can also appear. Echo, but no fetal signs [3]. (3) Incomplete abortion: The uterus enlarges or plump, the shape of the gestational sac is incomplete or collapses, there is no echo of the germ in the capsule, and no fetal heart beat occurs. This group of data in the clinical diagnosis of threatened abortion intended to prevent fetal treatment in 5 cases, B-ultrasound found that 2 cases of gestational sac less than gestational age, and deformation, no embryo and fetal heart beat, diagnosis is inevitable miscarriage, 1 case of intrauterine no see in the gestational sac, only the loose light band or the light-relief group was seen. The diagnosis was incomplete uterine cavity residue. Clinically, the treatment plan was promptly adjusted, and the curettage was performed. The pathological result of B-ultrasound was completely consistent with this. Obstetrics and Gynecology Ovarian tumors in acute abdomen torsion is also one of the causes of acute abdominal pain. When the ovarian tumor is torsioned, it can cause impediments to movement and vein blood supply, which can lead to extensive edema of the tumor and even death from blood. If there is a whirlpool sign, CDFI shows that there is no color flow signal around the mass and it can be diagnosed clearly [4]. The disease occurs in women and even children of any age. The sonogram shows that the affected side of the ovary disappears, and the cystic solid and mixed abnormal masses occur in the pelvic cavity and lower abdomen. The torsion is severe. The disease should be differentiated from the attached inflammatory mass and old ectopic pregnancy. It should be combined at the time of diagnosis. Medical history analysis. The rupture of the corpus luteum often has no history of amenorrhea, which occurs in 10 to 18 days of the menstrual cycle. It can also delay the menstruation. Ultrasound can be seen in the liquid dark area in the peritoneal cavity, and hypoechoic bolus can be seen in the attachment area. Transvaginal color Doppler ultrasound combined with blood HCG detection and posterior fistula puncture is a sensitive method for diagnosing rupture of the corpus luteum [5].

Acute pelvic inflammatory disease often has acute tubal inflammation, fallopian tube empyema, fallopian tube ovarian abscess, acute pelvic peritonitis. There are no special sonograms in the early stages of acute pelvic inflammatory disease. Frequent fever, increased white blood cells, and posterior fistula puncture are non-blood fluids. When there is a fallopian tube empyema or fallopian tube ovary abscess, the ultrasound image shows irregular masses on one side or both sides of the uterus. The mass has irregular borders, mostly cystic echo, poor sound transmission in the capsule, and weak light in the capsule. Point or floccular strong echo, unclear mass border, unclear boundary with ovary and fallopian tube, probe touched mass and pain, when there is a lot of exudate, a liquid dark area with poor permeability can be seen beside the uterus.

\section{Conclusion}

Obstetrics and Gynecology Acute abdominal disease is a common disease in women. Its main symptom is acute abdominal pain. With the rapid development of science and technology, the increasing precision of ultrasonic testing instruments and the continuous advancement of medical technology, the accuracy of ultrasound in the diagnosis of acute abdomen diseases in obstetrics and gynecology is significantly improved. Obstetrics and Gynecology acute abdomen have opportunities at any age, mainly in women of childbearing age, the cause of the disease is very 
complex, and the disease is fast, if you can not get rapid diagnosis and treatment, the consequences are very dangerous. Therefore, how to use ultrasound to quickly and accurately diagnose obstetric and gynecologic acute abdomen has become a hot topic in clinical medicine. The accuracy of ultrasound examination is high and rapid. According to the characteristics of ultrasound, the lesion location, pathological type, and the type and cause of acute abdomen can be diagnosed. The diagnosis can be assisted by the doctor to reduce the pain and the injury to the patient. To guide the clinical need for emergency surgery [20]. Since ultrasound has such a wide range of applications in the field of obstetrics and gynecology and acute abdomen and has such a large role, it can be used as the first-choice examination method for obstetrics and gynecology in acute abdomen and is worthy of further promotion in clinical practice.

\section{References}

[1] Ye Li. Clinical diagnosis and differential diagnosis of acute abdomen in obstetrics and gynecology[J]. Chinese Health Industry, 20 14, 1 6(2): 122 - 123.

[2] Huang Changju. Color Doppler ultrasound diagnosis of obstetric and gynecological acute abdomen [J]. Journal of Qiqihar Medical College, 20 14, 9 (1 0): 1465 - 1466.

[3] Chen Liju. Clinical analysis of ultrasound diagnosis of 70 cases of obstetric and gynecological acute abdomen [J]. Journal of Taishan Medical College, 20 14, 35 (1 0): 1066 - 1067.

[4] Huang Ning. Obstetrics and Gynecology acute abdomen ultrasound diagnosis [J]. Medical Theory and Practice, 201 0,1 (1 0): 89 - 90.

[5] Shen Haiyan. Obstetrics and Gynecology acute abdomen ultrasound diagnosis [J]. Medical Information (Middle-Term), 201 0, 12 (4): 3585 - 3586.

[6] Xu Ying. Ultrasonic diagnosis of acute abdomen in obstetrics and gynecology [J]. Practical Clinical Medicine, 200 9, 6 (8): 102 - 103. 